\title{
Design Tools for Random Sampling in Multistandard Radio Signal Processing
}

\author{
Khaled Grati, Manel Ben-Romdhane, Chiheb Rebai, Adel Ghazel \\ GRESCOM Research Lab, Sup'Com, University of Carthage \\ Cité Technologique El Ghazala Ariana, 2088, Tunisia \\ \{grati.khaled, manel.benromdhane, chiheb.rebai, adel.ghazel\}@supcom.tn \\ Patricia Desgreys, Patrick Loumeau \\ LTCI-CNRS UMR 5141, TELECOM ParisTech \\ 46, Rue Barrault, 75634 Paris Cedex 13, France \\ \{patricia.desgreys, patrick.loumeau\}@telecom-paristech.fr
}

\begin{abstract}
This paper proposes new extensions to random sampling analytical formulations suitable for radio signal spectral analysis. A Fourier transform spectrum estimator is established and verifies the alias-free condition independently of probability density functions for the additive random sampling scheme. Moreover, an energy spectrum density function formulation is proposed for an additive randomly sampled deterministic signal. The obtained theoretical results show random sampling alias suppression. Also, a time-quantized additive random sampling (TQ-ARS) based zero-padding spectral analysis tool is developed. Alias attenuations are computed to design the baseband stage of a TQ-ARS-based software defined radio multistandard receiver. Good performances are shown in terms of relaxing the anti-aliasing filter order, avoiding the automatic gain control, reducing the mean sampling frequency and reconstructing the quantized output spectrum.
\end{abstract}

Key words and phrases:Time-quantized additive random sampling; Spectrum analysis; Multistandard receiver; Anti-aliasing filter.

2010 AMS Mathematics Subject Classification -94A12, 94A20, 65C10, 65T50, 91A28, 94A05.

\section{Introduction}

Today, radio communication systems are facing new challenges in terms of quality of services, new functionalities, such as multimedia streaming, and seamless connection between different standards. Software radio (SWR) has been proposed as an emerging technology [22]. It allows a vertical handover on multiple 
communication standards (GSM, UMTS, WiFi, WiMAX, etc.) on a single network infrastructure [5]. An SWR receiver is not completely realizable, due to a technology bottleneck. Thus, the software defined radio (SDR) concept has been proposed [5]. The SDR receiver design approach attempts to place as much of the complex radio signal processing as possible into the digital domain. SDR receiver topology imposes severe constraints on the analog-to-digital conversion. Consequently, there are constraints on the dynamic range, sampling frequency and power consumption [5].

This paper proposes to randomly sample an analog radio signal with irregular time spacing instants, in order to reduce the constraints on analog-todigital conversion. Random sampling is called alias-free sampling, due to its alias suppression property. Random sampling schemes and related processing blocks (Fourier transform, filters, reconstruction algorithm, etc.) have been developed in previous works $[9,12,16,20,21,22,31]$. Generally, the used term is non-uniform sampling. It has been introduced in many areas, such as mathematics [20, 21], signal processing [33], image processing [23], geophysics and remote sensing [10], spectrum analyzers [2] and duty cycle correction [7]. In the literature, several non-uniform sampling techniques are proposed as regular time spacing with sample suppression, level-crossing sampling and random sampling.

The regular time spacing with sample suppression technique is used when the signal to be digitized varies within a short interval of time $[3,35]$. A common example is the electrocardiogram signal, where the shape of the peaks is more significant than the silent moment between two peaks. The authors in [35] presented an appropriate rate of sampling regarding the rate of changes of the signal. This technique reduces the data volume without losing valuable information. However, the regular time spacing with sample suppression technique is not suitable for radio signals since its shape is completely different from electrocardiogram signal.

The level-crossing sampling technique is usually used for asynchronous analogto-digital conversion $[1,15]$ and data compressive sensing $[19,27]$. This technique needs to previously define sampling levels. When the signal crosses preset amplitude levels, it triggers the sampling operation. This leads to a non-unifom sampling instants. The idea behind the compressive sensing is to make the redundancy removal closer to the signal sampling. When combined with sparse reconstruction algorithms, it could lead to good performances [27].

As far as it concerns the random sampling technique, there are well-described applications like duty cycle correction [7] and spectrum analyzers [2]. For the duty cycle correction, the authors in [7] introduced a novel idea based on a random sampling technique for measurement and local correction of the duty cycle of high-speed on-chip signals. The high measurement accuracy achievable through this random sampling technique provides a way to correct the duty cycle from $30 \%$ to $70 \%$. For spectrum analyzer application, the digital alias- 
free signal processing (DASP) technique was introduced in 1994. The DASP technique overcomes the uniform sampling limit formulated by the NyquistShannon sampling theorem, which stipulates that "if a function $x(t)$ contains no frequencies higher than $B$ cycles per second, it is completely determined by giving its ordinates at a series of points spaced $1 /(2 B)$ seconds apart" [25]. However, the DASP technique is not suitable for real-time embedded applications, such as radio communications. In fact, samples are collected and stored in a memory before spectral analysis and reconstruction.

Among non-uniform sampling techniques, the random sampling seems to be the most adapted technique with radio signals. Thanks to the alias-free property, this work considers the random sampling technique. The alias-free property preserves randomly sampled signal bandwidth from aliasing [8]. However, no complete analytical study and design methodology for a random sampling-based SDR multistandard receiver has been proposed in previous works.

In this paper, the authors present a review of random sampling theory and the analytical formulations in order to establish novel mathematical proofs. Then, the authors focus on the digitized signal spectrum to verify the alias attenuation propriety. The authors' contributions extend the analytical study of random sampling processing to practical concerns. Hence, the authors introduced the time-quantized random sampling scheme and estimate the alias attenuations for different cases. Consequently, the authors establish a random sampling-based SDR multistandard receiver design approach. This receiver takes advantage of random sampling to relax constraints on the analog-to-digital converter (ADC) or on the anti-aliasing filter (AAF) but also to avoid the use of automatic gain control (AGC) circuit.

Uniform sampling-based radio receiver designers usually have to optimize the trade-off between relaxing the constraints of the AAF, the AGC and the ADC. In fact, if the AAF is very selective and the signal is limited to the channel bandwidth, the sampling frequency of the ADC is limited to the Nyquist rate. If the AAF is relaxed and the signal is not limited only to the channel bandwidth, the ADC has to oversample the signal to avoid spectrum aliasing. Moreover, the analog bandwidth of the ADC includes less attenuated blockers, which limits AGC gain compression, leading to a higher ADC dynamic range.

In Section 2, a brief review of random sampling theory is presented, followed by the proposed novel mathematical proofs of alias-free digitized radio signals. Section 3 details the proposed formulation for the spectrum properties of a randomly sampled deterministic signal. In Section 4, a time-quantized additive random sampling (TQ-ARS) based zero-padding spectral analysis tool is defined, in order to allow the evaluation of spectral performances. TQ-ARS-based design considerations and results for an SDR multistandard radio receiver are presented in Section 5. Finally, conclusions and perspectives are drawn in section 6 . 


\section{Random Sampling Formulation and Novel Analy- tical Proof of its Alias-Free Property}

This section starts with a review of random sampling theory and the analytical formulations of schemes adapted to multistandard radio signals characteristics. A new Fourier transform spectrum estimator for signals is then developed, in order to establish novel mathematical proofs of the digitized signal alias-free spectrum.

\subsection{Review of non-uniform sampling analytical formulation}

The random sampling process converts a continuous analog bandpass signal, $x(t)$, into its discrete $x_{s}(t)$ representation, as given by (1),

$$
x_{s}(t)=x(t) s(t)=x(t) \sum_{k=-\infty}^{+\infty} \delta\left(t-t_{k}\right)=\sum_{k=-\infty}^{+\infty} x\left(t_{k}\right) \delta\left(t-t_{k}\right)
$$

where $s(t)$ is the sampling function.

The sampling instants sequence, $\left\{t_{k}\right\}$, is defined as $t_{k-1}<t_{k}$, where $\left\{t_{k}\right\}$ is a set of independent and identically distributed (i.i.d.) random variables with $\left\{k T_{s}\right\}$ as the mean values and $T_{s}$ as the mean sampling period. The instants, $t_{k}$, can be considered as points on the time axis [8]. The sampling instants sequence, $\left\{t_{k}\right\}$, represents a point process [4].

The sampling point density function [8] is defined as follows: Let $p_{k}(t)$ be the probability density functions of time intervals, $\left(t_{k}-t_{0}\right)$, for $k \in \mathbb{N}^{*}$, where $0 \leq t_{0}<t_{1}<t_{2}<\cdots<t_{k}<\cdots<t_{n}$ with $\lim _{n \rightarrow+\infty} t_{n}=+\infty$. The sampling point density function, $p(t)$, is defined by (2).

$$
p(t)=\sum_{k=0}^{+\infty} p_{k}(t)
$$

The sampling point density function has to be of the form shown in (3) to carry out the alias-free sampled signal at the mean sampling frequency, $f_{s}$. Function $p(t)$ tends to become constant when $t$ exceeds a delay, $T_{a}$, where $T_{a}$ depends on the probability density functions, $p_{k}(t)[8]$.

$$
\left.p(t)\right|_{t \geq T_{a}}=f_{s}=1 / T_{s}
$$

Under the alias-free condition (3), random sampling is known as alias-free sampling. Alias-free sampling is sampling while suppressing the uniform sampling replicas at $\left(f \pm k f_{s}\right)$ with $f$ as the signal frequency and $f_{s}$ as the sampling frequency.

Different random sampling schemes have been proposed $[1,8,33]$, but the most widely used ones are the jittered random sampling (JRS) and additive 
random sampling (ARS) schemes. The infinity of values of sampling instants distributed in the intervals, $\left[k T_{s},(k+1) T_{s}\right]$, does not allow for the practical implementation of the JRS and ARS schemes. To overcome this limitation, Wojtiuk proposed the TQ-ARS scheme [33]. In this paper, the TQ-ARS scheme is considered for the proposed spectral analysis tool and for application in the design of novel multistandard radio receiver architecture. This section continues by presenting random sampling scheme formulations before developing the proposed novel proofs for alias-free property.

\subsubsection{Jittered random sampling scheme}

Jittered random sampling is known as periodic sampling with a deliberate jitter. This scheme is modeled by (4) where $\left\{\tau_{k}\right\}$ is a set of i.i.d. random variables with zero mean [8],

$$
t_{k}=k T_{s}+\tau_{k}, k \in \mathbb{N}^{*}
$$

with $E\left[t_{k}\right]=k T_{s}$ and $\operatorname{Var}\left(t_{k}\right)=\sigma^{2}$.

Let $t_{0}=0$ with a probability density function of $p_{0}(t)=\delta(t)$. The aliasfree property of the JRS scheme is verified only for uniform probability density functions, $p_{k}(t)$, over $\left[k T_{s}-T_{s} / 2, k T_{s}+T_{s} / 2\right]$. In fact, the alias-free condition of (3) is satisfied by construction where functions $p_{k}(t)$ are equal to $1 / T_{s}$. Thus, the sampling point density function, $p(t)$, the sum of the $p_{k}(t)$, is equal to $1 / T_{s}$ when $t$ exceeds $T_{s} / 2$. In addition, the root mean square, $\sigma$, is equal to $(1 / \sqrt{12}) T_{s}$. Let $\sigma / T_{s}$ be the statistical parameter and $1 / \sqrt{12}$ be its highest value satisfying the increasing sampling instants, $t_{k}$.

\subsubsection{Additive random sampling scheme}

Equation (5) represents an analytical model of the additive random sampling scheme for an initial instant, $t_{0}[8]$,

$$
t_{k}=t_{k-1}+\tau_{k}=t_{0}+\sum_{i=1}^{k} \tau_{i}, k \in \mathbb{N}^{*}
$$

with $E\left[t_{k}\right]=k T_{s}$ and $\operatorname{Var}\left(t_{k}\right)=k \sigma^{2}$, where $\left\{\tau_{k}\right\}$ is a set of i.i.d. random variables with a probability density function of $p_{1}(t)$, a mean $E\left[\tau_{k}\right]=T_{s}$ and a variance $\operatorname{Var}\left(\tau_{k}\right)=\sigma^{2}$. Let $t_{0}=0$ with a probability density function of $p_{0}(t)=\delta(t)$. For $k \neq 0$, the probability density function of the $k^{t h}$ sampling instant is given by $(6)$,

$$
p_{k}\left(t_{k}\right)=\circledast_{i=1}^{k} p_{1}\left(\tau_{i}\right)
$$

where $\circledast$ denotes the convolution product. 


\subsubsection{Time-quantized random sampling scheme}

For practical concerns [18], JRS and ARS instants should be quantized. In the time-quantized random sampling (TQ-RS) scheme, as illustrated in Figure 1 , the time is quantized in instants that are multiples of a step, $\Delta$, given by (7) [33] where $q_{T}$ is the time quantization factor. The step $\Delta$ should be relatively small compared to the mean sampling period, $T_{s}$. The sampling instant, $t_{k, q}$, is achieved with (8) for the random instant, $t_{k}$. The time between two consecutive random instants is $\delta t_{k}=t_{k}-t_{k-1}$. The time between two consecutive time-quantized random instants is $\delta t_{k, q}=t_{k, q}-t_{k-1, q}$. Wojtiuk concluded that, with $\Delta=T_{s} / 10$ for the TQ-RS based on ARS scheme, the obtained power spectrum density (PSD) of the sampled signal is similar to the results of the ARS scheme [33].

$$
\begin{aligned}
& \Delta=\frac{T_{s}}{q_{T}} \\
& \delta t_{k, q}=n \Delta i f\left(n-\frac{1}{2}\right) \Delta<\delta t_{k} \leq\left(n+\frac{1}{2}\right) \Delta \\
& \text { || } k T_{s}: k^{\text {th }} \text { uniform sampling instant } \\
& \text { o } t_{k}: k^{\text {th }} \text { random instant (ARS or JRS scheme) } \\
& \times \text { Possibilities for time-quantized instants } \\
& \curvearrowright \text { From } t_{k} \text { to } t_{k, q}: k^{\text {th }} \text { time-quantized random instant }
\end{aligned}
$$

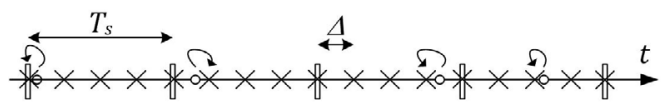

Figure 1: Time-quantized random sampling scheme description for $q_{T}=4$.

\subsection{Novel proofs of alias-free digitized signal}

Bilinskis proved in [8] that, if condition (3) is fulfilled, the spectrum of the digitized signal is alias free. Masry proposed in [21] Fourier transform estimates for antithetical stratified random sampling. However, in this paper, the provided proofs need a new Fourier transform spectrum estimator for the randomly sampled signals. This Fourier transform spectrum estimator corresponds to the mean of the randomly sampled signal spectrums and allows more convenient formulations for non-uniformly sampled signals.

\subsubsection{Proposed general proof formulation of alias-free property}

Let $x(t)$ be the input signal and $x_{s}(t)$ be the output signal of the sampler given by (1), each sample, $x\left(t_{k}\right)$, corresponds to (9). A new Fourier transform estimation of $x_{s}(t)$ is proposed and given by $(10)$. 


$$
\begin{aligned}
& x\left(t_{k}\right)=\int_{-\infty}^{+\infty} x(t) \delta\left(t-t_{k}\right) d t \\
& \hat{X}_{s}(f)=E\left[\sum_{k=-\infty}^{+\infty} x\left(t_{k}\right) e^{-j 2 \pi f t_{k}}\right] \\
&=E\left[\sum_{k=-\infty}^{+\infty}\left[\int_{-\infty}^{+\infty} x(t) \delta\left(t-t_{k}\right) d t\right] e^{-j 2 \pi f t_{k}}\right] \\
&=E\left[\sum_{k=-\infty}^{+\infty}\left[\int_{-\infty}^{+\infty} x(t) \delta\left(t-t_{k}\right) e^{-j 2 \pi f t_{k}} d t\right]\right] \\
&=E\left[\sum_{k=-\infty}^{+\infty}\left[\int_{-\infty}^{+\infty} x(t) \delta\left(t-t_{k}\right) e^{-j 2 \pi f t} d t\right]\right]
\end{aligned}
$$

Definition of measurable space, $\left(W^{\infty}, \mathbb{R}^{\infty}, P^{\infty}\right)[28]$ : Let $\left\{t_{k}, k \in \mathbb{Z}\right\}$ be a finite dimension random variables defined over a measurable space, $\left(W^{\infty}, \mathbb{R}^{\infty}, P^{\infty}\right)$, with probability density functions in $\left\{p_{k}(t), k \in \mathbb{Z}\right\}$, where $W^{\infty}$ is defined in (11),

$$
W^{\infty}=\cdots \times W_{0} \times W_{1} \times W_{2} \times \cdots \times W_{i} \times \cdots
$$

where $W_{i}=W=\mathbb{R}, i \in \mathbb{Z}$.

Definition of marginal probability on $W_{k}^{\infty}$ [28]: Let $W_{k}^{\infty}$ be the space, $W^{\infty}$, without the $k^{t h}$ coordinate. The marginal probability is defined by (12).

$$
d P_{k}^{(1)}=\int_{W_{k}^{\infty}} d P^{\infty}
$$

Definitions (11) and (12) enable us to obtain (13) from (10).

$$
\begin{aligned}
\hat{X}_{s}(f) & =\int \cdots \int_{W^{\infty}} \sum_{k=-\infty}^{+\infty}\left[\int_{-\infty}^{+\infty} x(t) \delta\left(t-t_{k}\right) e^{-j 2 \pi f t} d t\right] d P^{\infty} \\
& =\int_{W} \sum_{k=-\infty}^{+\infty}\left[\int_{-\infty}^{+\infty} x(t) \delta\left(t-t_{k}\right) e^{-j 2 \pi f t} d t\right] d P_{k}^{(1)} \\
& =\sum_{k=-\infty}^{+\infty} \int_{-\infty}^{+\infty} \int_{W} x(t) \delta\left(t-t_{k}\right) e^{-j 2 \pi f t} d P_{k}^{(1)} d t
\end{aligned}
$$

As $p_{k}(t)$ is the probability density function of the time interval, $\left(t_{k}-t_{0}\right),(13)$ leads to (14). 


$$
\begin{aligned}
\hat{X}_{s}(f) & =\sum_{k=-\infty}^{+\infty} \int_{t=-\infty}^{+\infty}\left[\int_{t_{k}=-\infty}^{+\infty} x(t) \delta\left(t-t_{k}\right) e^{-j 2 \pi f t} p_{k}\left(t_{k}\right) d t_{k}\right] d t \\
& =\sum_{k=-\infty}^{+\infty} \int_{t=-\infty}^{+\infty} x(t) e^{-j 2 \pi f t}\left[\int_{t_{k}=-\infty}^{+\infty} \delta\left(t-t_{k}\right) p_{k}\left(t_{k}\right) d t_{k}\right] d t \\
& =\sum_{k=-\infty}^{+\infty} \int_{t=-\infty}^{+\infty} x(t) e^{-j 2 \pi f t} p_{k}(t) d t \\
& =\int_{t=-\infty}^{+\infty} \sum_{k=-\infty}^{+\infty} x(t) e^{-j 2 \pi f t} p_{k}(t) d t \\
& =\int_{t=-\infty}^{+\infty} x(t) e^{-j 2 \pi f t} \sum_{k=-\infty}^{+\infty} p_{k}(t) d t \\
& =\int_{t=-\infty}^{+\infty} x(t) e^{-j 2 \pi f t} p(t) d t
\end{aligned}
$$

Considering the alias-free condition of (3) and substituting $1 / T_{s}$ for $p(t)$ into (14) yields (15), where $X(f)$ is the continuous Fourier transform of $x(t)$.

$$
\hat{X}_{s}(f)=\frac{1}{T_{s}} \int_{t=-\infty}^{+\infty} x(t) e^{-j 2 \pi f t} d t=\frac{1}{T_{s}} X(f)
$$

Thanks to this new formulation, the estimation $\hat{X}_{s}(f)$ is proportional to the Fourier transform of $x(t)$, unlike the uniform scheme where $X_{s}(f)$ is the sum of the replicas of $X(f)$ at the frequencies, $f \pm k f_{s}, k \in \mathbb{Z}[26]$. This means that, if condition (3) is satisfied, the sampled signal is free from aliasing, i.e., alias free.

\subsubsection{Proposed proof formulation of ARS alias-free condition}

In the case of the ARS scheme (5), $t_{k}$ is the sum of $k$ i.i.d. random variables $\left(\tau_{1}, \tau_{2}, \ldots, \tau_{k}\right)$ with a mean of $T_{s}$, a standard deviation of $\sigma$ and a probability density function of $p_{k}(t)$. The central limit theorem proves that $p_{k}(t)$ converges to a Gaussian distribution when $k$ tends to infinity, as given in (16).

$$
t_{k}=\sum_{i=1}^{k} \tau_{i} \stackrel{D}{\rightarrow} \mathcal{N}\left(k T_{s}, \sqrt{k \sigma^{2}}\right)
$$

The sampling point density function, $p(t)$, yields to an explicit formula as a series of Gaussian distributions, $\mathcal{N}\left(k T_{s}, \sqrt{k \sigma^{2}}\right)$, as given by (17).

$$
p(t)=\sum_{k=1}^{+\infty} \frac{1}{\sqrt{2 \pi k \sigma^{2}}} e^{-\frac{\left(t-k T_{s}\right)^{2}}{2 k \sigma^{2}}}
$$


The characteristic function of a Gaussian distribution $\mathcal{N}(m, s)$ is written as (18); then, the characteristic function of $p(t)$ is obtained in (19).

$$
\begin{gathered}
\Phi_{X}(-f)=\mathcal{T} \mathcal{F}\left[p_{X}(t)\right]=e^{-j 2 \pi f m} e^{-2 \pi^{2} s^{2} f^{2}} \\
\Phi(-f)=\mathcal{T} \mathcal{F}[p(t)]=\sum_{k=1}^{+\infty}\left(e^{-j 2 \pi f T_{s}-2 \pi^{2} \sigma^{2} f^{2}}\right)^{k}
\end{gathered}
$$

For $f>0$, the geometric series with the general term $q=e^{\left(-j 2 \pi f T_{s}-2 \pi^{2} \sigma^{2} f^{2}\right)}$ converges. Since $|q|<1$, the $p(t)$ Fourier transform is expressed as in (20).

$$
\mathcal{T} \mathcal{F}[p(t)]=\frac{1}{e^{j 2 \pi f T_{s}+2 \pi^{2} \sigma^{2} f^{2}}-1}
$$

The sampling point density function limit (21), when $t$ tends to infinity, follows from applying the final value theorem.

$$
\lim _{t \rightarrow+\infty} p(t)=\lim _{f \rightarrow 0^{+}} j 2 \pi f \mathcal{T} \mathcal{F}[p(t)]=\lim _{f \rightarrow 0^{+}} \frac{j 2 \pi f}{j 2 \pi f T_{s}+2 \pi^{2} \sigma^{2} f^{2}}=\frac{1}{T_{s}}
$$

Equations (17) and (21) show $p(t)$ convergence to the alias-free condition given in (3). Therefore, the ARS scheme satisfies the alias-free condition independently of the distributions of $p_{k}(t)$, especially with all configurations of uniform and Gaussian distributions. Figure 2 illustrates this behavior for a Gaussian distribution with a statistical parameter of $\sigma / T_{s}=0.15$. The given example shows that $p(t)$ tends to $10 \mathrm{~Hz}$ for $T_{s}=0.1 \mathrm{~s}$ after a delay $T_{a}$ near to 8 times $T_{s}$.

The ARS scheme is, therefore, more interesting to study than the JRS scheme as all distributions can be used and satisfies the alias-free condition.

\section{Formulation of Spectrum Proprieties of Randomly Sampled Deterministic Signals}

In the previous section, the authors considered that all the sampling instants were totally random, in order to verify the alias-free property. However, in radio communications, the first instant is defined as a deterministic initial time. In the literature, only randomly sampled random signals were studied. Wojtiuk established the power spectrum density functions of a randomly sampled signal using both the JRS and ARS schemes [33]. In this section, the authors propose to extend Wojtiuk's work by considering an additive randomly sampled deterministic signal. A new analytical formulation of the energy spectrum density (ESD) function is established. Deterministic signal are often used for ADC test setup. 

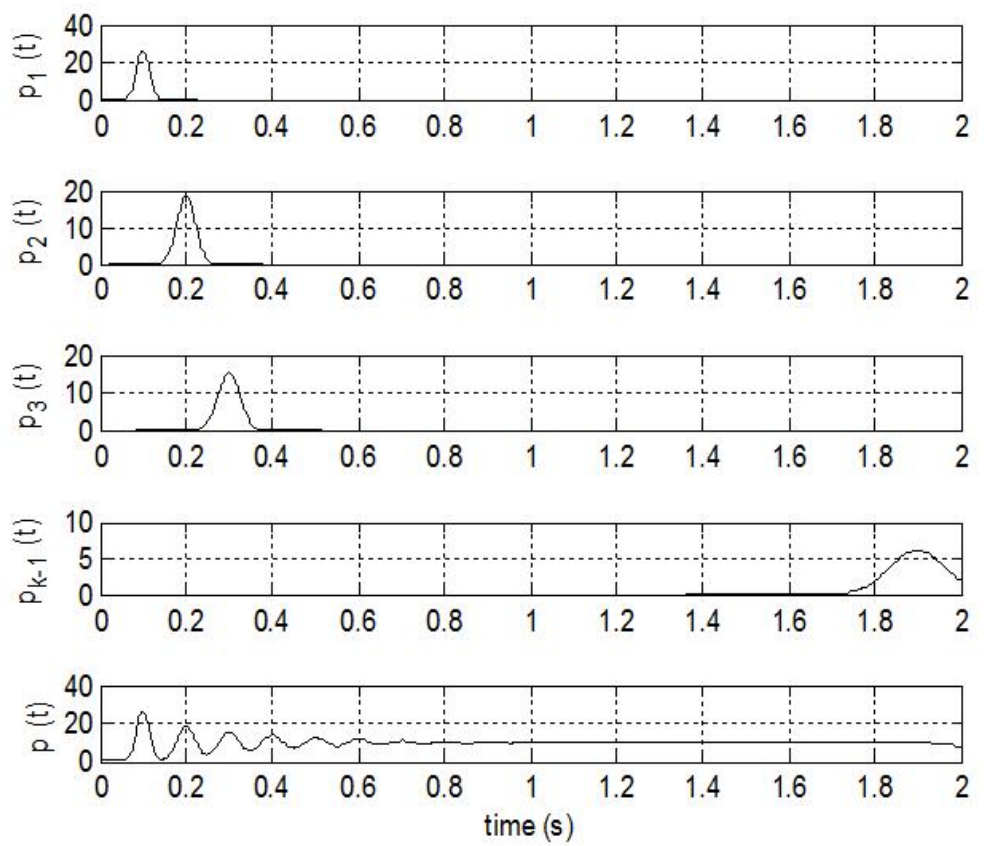

Figure 2: ARS sampling point density function for Gaussian distribution with $T_{s}=0.1 s, \sigma / T_{s}=0.15$ and $k=20$.

In fact, sine-wave signals are generally applied in the preliminary experimental validation. The Fourier transform estimation of an additive randomly sampled signal is composed of wideband noise added to the original signal. To develop a formula of the ESD function, the authors define a random variable, $T_{k}$, similarly to the random variable $\tau_{k}$ in the JRS scheme defined in (4). Equation (22) follows the ARS definition (5).

$$
\begin{aligned}
T_{k} & =t_{k}-k T_{s} \\
& =t_{k-1}+\tau_{k}-k T_{s} \\
& =t_{0}+\sum_{i=1}^{k}\left(\tau_{i}-T_{s}\right)
\end{aligned}
$$

Let, for $k \neq 0, \tau_{k}^{\prime}=\tau_{k}-T_{s}$ be a random variable, then $T_{k}=\sum_{i=1}^{k} \tau_{i}^{\prime}$. The densities, $\tilde{p}_{k}(T)$ and $\tilde{p}_{1}\left(\tau^{\prime}\right)$, are given respectively in (23) and are equal to the $p_{k}(t)$ translated with $k T_{s}$ and the $p_{1}(\tau)$ translated with $T_{s}$.

$$
\begin{array}{r}
\tilde{p}_{k}\left(T_{k}\right)=\tilde{p}_{k}\left(t_{k}-k T_{s}\right)=p_{k}\left(t_{k}\right) \\
\tilde{p}_{1}\left(\tau_{i}^{\prime}\right)=\tilde{p}_{1}\left(\tau_{i}-T_{s}\right)=p_{1}\left(\tau_{i}\right)
\end{array}
$$


Therefore, according to (6), the $T_{k}$ probability density function, $\tilde{p}_{k}\left(T_{k}\right)$, leads to $(24)$.

$$
\tilde{p}_{k}\left(T_{k}\right)=\circledast_{i=1}^{k} \tilde{p}_{1}\left(\tau_{i}^{\prime}\right)
$$

For this new random variable, $T_{k}$, the mean of the sampling function given in (1) results in (25),

$$
\begin{aligned}
E[s(t)] & =\sum_{k=0}^{+\infty} \int_{\mathbb{R}} \delta\left(t-k T_{s}-T_{k}\right) \tilde{p}_{k}\left(T_{k}\right) d T_{k} \\
& =\delta(t)+\sum_{k=1}^{+\infty} \tilde{p}_{k}\left(t-k T_{s}\right) \\
& =\delta(t)+\sum_{k=1}^{+\infty} \tilde{p}_{k}(T)
\end{aligned}
$$

with $T=t-k T_{s}$. For $k \neq 0$, the $\tilde{p}_{k}(T)$ Fourier transform is written in (26).

$$
\begin{aligned}
\mathcal{T F}\left[\tilde{p}_{k}(T)\right] & =\mathcal{T} \mathcal{F}\left[\circledast_{i=1}^{k} \tilde{p}\left(\tau^{\prime}\right)\right] \\
& =\prod_{i=1}^{k} \mathcal{T} \mathcal{F}\left[\tilde{p}_{1}\left(\tau^{\prime}\right)\right] \\
& =\prod_{i=1}^{k} \mathcal{T} \mathcal{F}\left[p_{1}(\tau)\right] \\
& =\prod_{i=1}^{k} \Phi_{1}(-f)
\end{aligned}
$$

The Fourier transform estimation is given in (27). As the characteristic function is always equal to 1 for $f=0$ and also verifies the propriety that $\left|\Phi_{1}(-f)\right|<1$ forf $>0$, the authors assume that the characteristic function corresponds to (28).

$$
\begin{aligned}
\hat{X}_{s}(f) & =X(f) \circledast\left[1+\sum_{i=1}^{+\infty}\left[\Phi_{1}(-f)\right]^{k}\right] \\
& =X(f) \circledast \sum_{i=0}^{+\infty}\left[\Phi_{1}(-f)\right]^{k}
\end{aligned}
$$




$$
\begin{aligned}
\Phi_{1}(-f) & =\left\{\begin{array}{l}
\Phi_{1}^{\prime}(r, f), f>0 \\
\lim _{f \rightarrow 0} \Phi_{1}^{\prime}(1, f)
\end{array}\right. \\
\text { where } \Phi_{1}^{\prime}(r, f) & =\left\{\begin{array}{l}
r e^{j 2 \pi f T_{s}}, 0<r<1 \\
e^{j 2 \pi f T_{s}}, r=1
\end{array}\right.
\end{aligned}
$$

For $f>0$, the Fourier transform estimation (27) represents the wideband noise (29). However, when $f$ tends to zero, the Poisson formula in (30) yields to the Fourier transform estimation equal to the original signal (31).

$$
\begin{gathered}
\hat{X}_{s}(f)=X(f) \circledast \sum_{i=0}^{+\infty} r^{k} e^{j 2 \pi f k T_{s}} \\
=X(f) \circledast \frac{1}{1-r e^{j 2 \pi f T_{s}}} \\
=X(f) \circledast \frac{1}{1-\Phi_{1}(-f)} \\
\sum_{i=0}^{+\infty} e^{j 2 \pi f k T_{s}}=\frac{1}{T_{s}} \sum_{i=0}^{+\infty} \delta\left(f-\frac{k}{T_{s}}\right) \\
\hat{X}_{s}(f)=\frac{1}{T_{s}} X(f) \circledast \lim _{f \rightarrow 0} \sum_{i=0}^{+\infty} \delta\left(f-\frac{k}{T_{s}}\right) \approx \frac{1}{T_{s}} X(f)
\end{gathered}
$$

Finally, the authors considered both cases in (29) and (31) to obtain the Fourier transform estimation in (32). The mean sampling period is $T_{s}$, the characteristic function of the probability density function, $p_{1}(\tau)$, is $\Phi_{1}(f)$, and the continuous-time Fourier transform of the analog signal, $x(t)$, is $X(f)$.

$$
\hat{X}_{s}(f)=X(f) \circledast \frac{1}{1-\Phi_{1}(-f)}+\frac{1}{T_{s}} X(f)
$$

The Fourier transform estimation in (32) contains not only the Fourier transform of $x(t)$ as in (15), but also wideband noise depending on the chosen probability density function. The wideband noise is due to the first deterministic sampling instant, $t_{0}$. In the case of uniform distribution over $\left[(1-a) T_{s},(1+a) T_{s}\right]$ with $0<a \leq 0.5$, the characteristic function is written as in (33).

$$
\Phi_{1}(f)=e^{j 2 \pi f T_{s}} \operatorname{sinc}\left(2 \pi f a T_{s}\right)
$$

The energy spectrum density is equal to the squared modulus of the Fourier transform estimation, $\left|\hat{X}_{s}(f)\right|^{2}$. Figure 3 shows the normalized ESD for the uniform distribution of $p_{1}(\tau)$ over $\left[T_{s} / 2,3 T_{s} / 2\right]$. It verifies the alias attenuation. 
In fact, Figure 3 illustrates an attenuation of quite $60 \mathrm{~dB}$ near $f_{s}$. In the case of a Gaussian distribution with a mean of $T_{s}$ and a variance of $\sigma^{2}$, the characteristic function (18) becomes (34).

$$
\Phi_{1}(f)=e^{j 2 \pi f T_{s}} e^{-2 \pi^{2} \sigma^{2} f^{2}}
$$

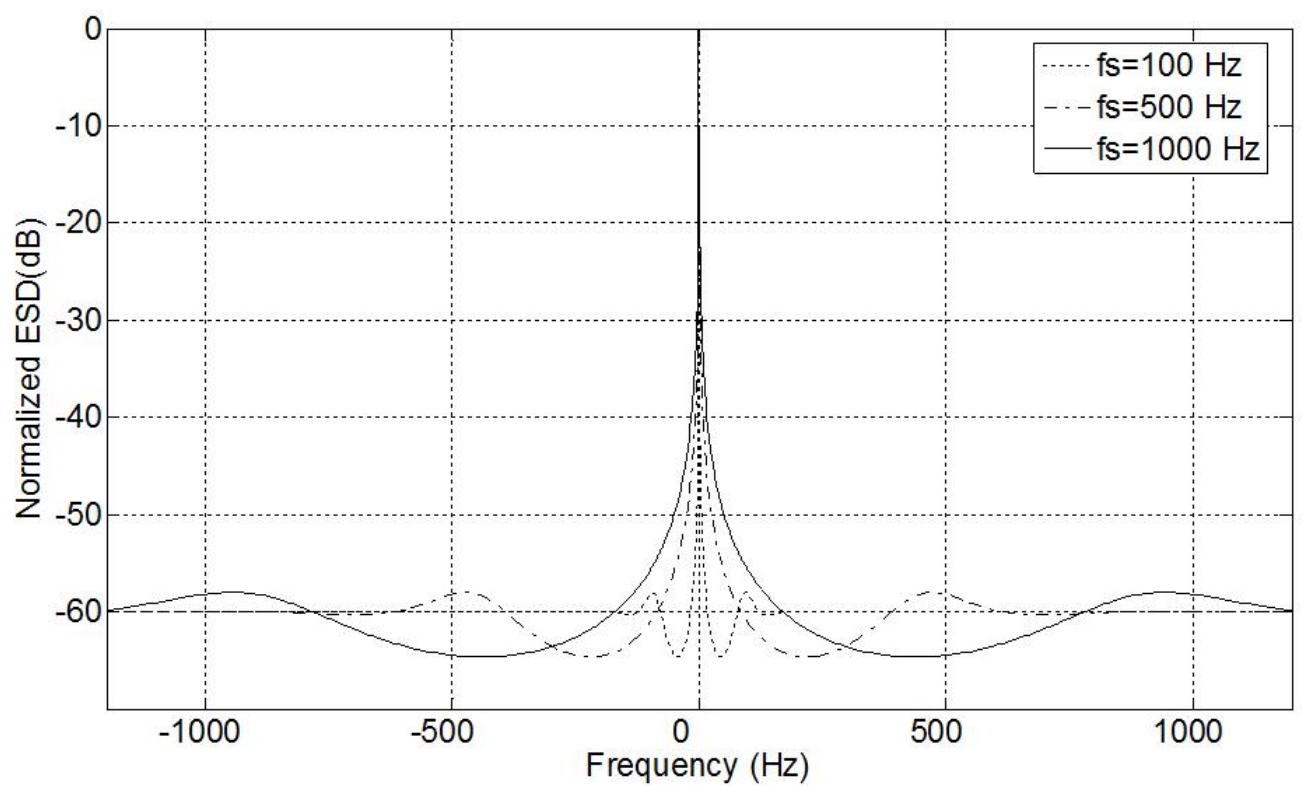

Figure 3: ESD of additive randomly sampled signal with the $1^{\text {st }}$ sample uniform distribution over $\left[T_{s} / 2,3 T_{s} / 2\right]$.

The normalized ESD for Gaussian distribution with $\sigma / T_{s}=1 / \sqrt{12}$ is presented in Figure 4. It shows better performances in terms of alias attenuation compared to the results of normalized ESD for the uniform distribution in Figure 3. In fact, there is quite $120 \mathrm{~dB}$ attenuation near $f_{s}$. This difference in attenuation is due to the wideband noise term in (32). This term depends on $\Phi_{1}(f)$ given in (33) for uniform distribution and in (34) for Gaussian distribution.

Equations (15) and (32) are obtained with sampling at infinite random instants and at infinite random instants starting from a deterministic instant $t_{0}$, respectively. However, in this paper, the authors are interested in suitable random instants for implementation. The signal is randomly sampled at finite time-quantized additive random instants. Therefore, a spectral analysis tool is proposed to show the impact of finite time-quantized additive random samples on the obtained spectrum and to verify replica suppression or evaluate replica attenuation. 


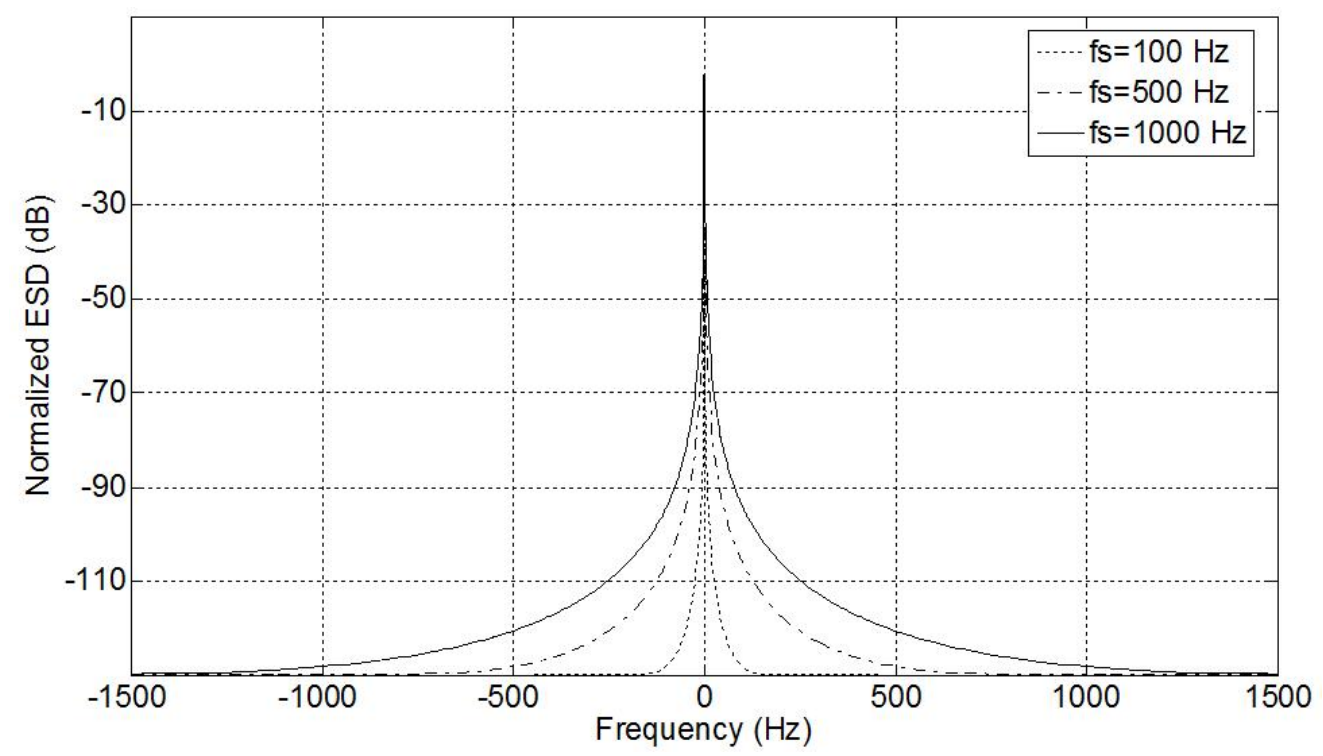

Figure 4: ESD of the additive randomly sampled signal with a Gaussian distribution for the statistical parameter $\sigma / T_{s}=1 / \sqrt{12}$.

\section{Definition of a TQ-RS-Based Zero-Padding Spec- tral Analysis Tool}

A spectral analysis tool is required to evaluate the alias suppression or attenuation of the time-quantized randomly sampled deterministic signal. Several studies focus on spectral analysis tools suitable for non-uniformly sampled signals. As explained in the following subsection, these tools present some drawbacks. Hence, after a brief analysis, the authors propose a new spectrum analysis tool suitable for the TQ-RS scheme, especially for time-quantized additive random sampling (TQ-ARS) scheme.

\subsection{Analysis of existing techniques for non-uniform spectral ana- lysis}

Discrete Fourier transform (DFT) estimation is achieved by three methods [9]. The first method, the point rule non-uniform transform discrete Fourier transform (NUT-DFT), is the conventional discrete Fourier transform that is appropriate for random sampling instants. The second method is based on interpolation, either the trapezoidal rule NUT-DFT, which involves a linear interpolation, or the Simpson's rule NUT-DFT, which involves a second-order interpolation. The third method considers the use of a "semi-analytic method", which is useful when the samples are logarithmically spaced. These methods are nearly equiva- 
lent. Indeed, these spectral analysis methods suffer from a relatively high-noise floor, due to a non-uniform sampling process [9]. The second drawback is the spectral analysis representation which is only within $\left[0, f_{s} / 2\right]$ with $f_{s}$ as the mean sampling frequency.

Another theoretical method for spectral analysis uses the non-uniform DFT to generalize the conventional DFT for the non-uniform sampling process [20]. However, this spectral analysis needs to be completed, in order to represent non-uniform sampled signal spectrum. The DASP team introduced a different method for spectral analysis [2]. This method applies a zero padding and then an iterative approach for the spectral analysis and signal reconstruction. Each step requires a threshold definition, new estimation values of samples and an inverse DFT computation. Moreover, this spectral analysis, which achieves reconstruction, requires at least 10 iterations and, therefore, involves a high computational cost.

\subsection{Proposed TQ-ARS-based zero-padding spectral analysis tool}

In the case of uniform sampling, fast Fourier transform (FFT) based spectral analysis represents the spectrum over a $\left[0, f_{s} / 2\right]$ band with $f_{s}$ as the sampling frequency. In the case of random sampling, the spectrum may be represented from the direct component (DC) frequency up to infinity, since the inter-sample time spacing tends towards zero. The existing spectral analyses $[5,9,20]$ present some computational constraints, require a signal reconstruction, or represent a spectrum over $\left[0, f_{s} / 2\right]$ for a mean sampling frequency equal to $f_{s}$. Consequently, the authors propose a new spectral analysis tool based on zero padding that is suitable for the TQ-ARS scheme. For $N$-length TQ-ARS instants, $\left\{t_{k, q}, 1 \leq k \leq\right.$ $N\}$, as constructed in (8), this tool follows three steps:

1. Sampling the test signal at instants of $t_{k, q}$ to obtain the $N$ samples $x_{k, q}$ for $1 \leq k \leq N$,

2. Padding zeros at non-used possibilities of time-quantized instants at a multiple of $\Delta$, as shown in Figure 1, to obtain $q_{T} N$ points. When using $q_{T} N$ points, it becomes possible to represent the spectrum over $\left[0, q_{T} f_{s} / 2\right]$,

3. Computing the FFT on the obtained $q_{T} N$ points to represent the spectrum between the DC and $q_{T} f_{s} / 2$ frequencies. Applying the DFT on the $N$ samples $x_{k, q}$ returns exactly the same values between $\mathrm{DC}$ and $f_{s} / 2$ as using zero-padding and FFT on the $q_{T} N$ points.

This simple tool is more suitable for a single-tone signal to estimate the alias attenuation level. For $\sigma / T_{s}=1 / \sqrt{12}$ and $q_{T}=16$, a $1.3 \mathrm{MHz}$ single-tone signal is time-quantized additive randomly sampled at a mean sampling frequency of $3.125 \mathrm{MHz}$, slightly greater than the Nyquist frequency. The obtained spectrum is presented in Figure 5 . 


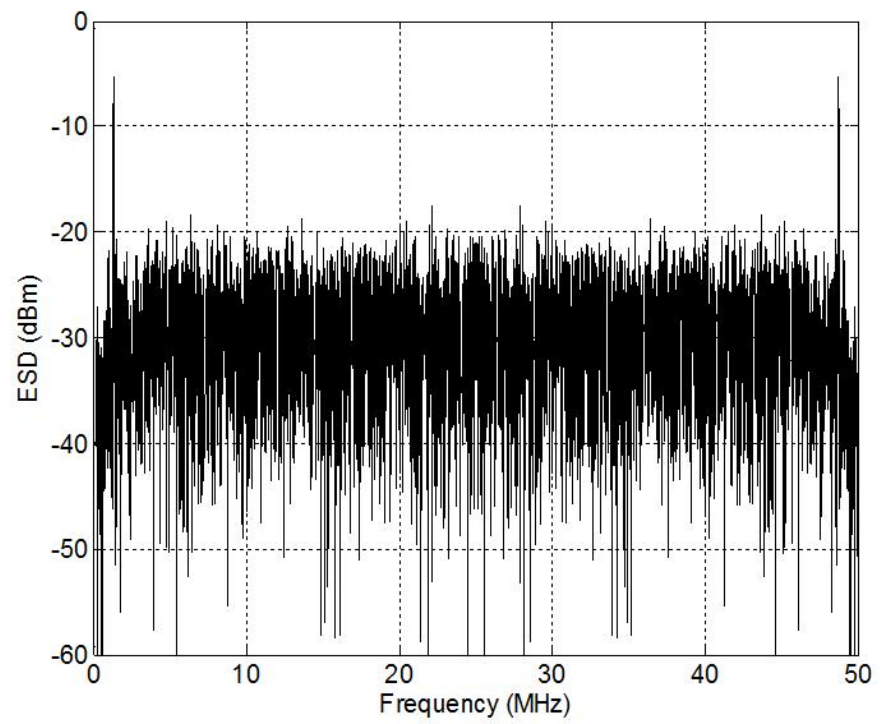

Figure 5: TQ-ARS-based zero-padding spectral analysis for $q_{T}=16$.

\subsection{Spectrum profiling}

To obtain a more accurate spectral analysis, the authors propose the computation of the mean spectral analysis relative to different generated time-quantized additive random instants $\left\{t_{k, q}, 1 \leq k \leq N\right\}$. In fact, the attenuations in successive bands $\left\{\left[(2 m-1) f_{s} / 2,(2 m+1) f_{s} / 2\right], 1 \leq m \leq\left(q_{T}-1\right) / 2\right\}$, depend on the generated time-quantized additive random instants. The mean of these attenuations, as presented in Figure 6, are evaluated with a mean spectral analysis relative to one hundred different sequences $\left\{t_{k, q}\right\}$ for statistical parameters of $\sigma / T_{s}$ equal to $0.05,0.1$ and 0.3 . The authors generated one hundred different sequences to correctly measure the attenuation value of aliases.

For the single-tone input frequency, $f_{i n}$, the mean sampling frequency of $f_{s}$, as $f_{\text {in }}<f_{s} / 2$ or $f_{s} / 2<f_{\text {in }}<q_{T} f_{s} / 2$, the alias attenuation amount is exactly the same. Therefore, randomly sampled signals can be up to $q_{T} f_{s} / 2$ instead of $f_{s} / 2$ for uniformly sampled signals, where the signal frequencies must be up to the Nyquist frequency. According to Figure 6, the aliases in bands $\left\{\left[(2 m-1) f_{s} / 2,(2 m+1) f_{s} / 2\right], 1 \leq m \leq\left(q_{T}-1\right) / 2\right\}$, depend only on the $\sigma / T_{s}$ parameter. Hence, in the case of a random sampling process, the alias attenuation, $A t t_{T Q-A R S_{m}}$, is defined as the attenuation of the replica in $\left[(2 m-1) f_{s} / 2,(2 m+1) f_{s} / 2\right]$ regarding to the single-tone signal power.

Figure 7 illustrates the profile of attenuations $\left\{A t t_{T Q-A R S_{m}}, 1 \leq m \leq\right.$ $\left.\left(q_{T}-1\right) / 2\right\}$ for different values of the statistical parameter, $\sigma / T_{s}$, and the oversampling ratio (OSR), where OSR is the mean sampling frequency $f_{s}$ out of the Nyquist frequency, which corresponds here to $2 f_{\text {in }}$. Figure 7 shows that 
DESIGN TOOLS FOR RANDOM SAMPLING IN MULTISTANDARD RADIO SIGNAL PROCESSING 133
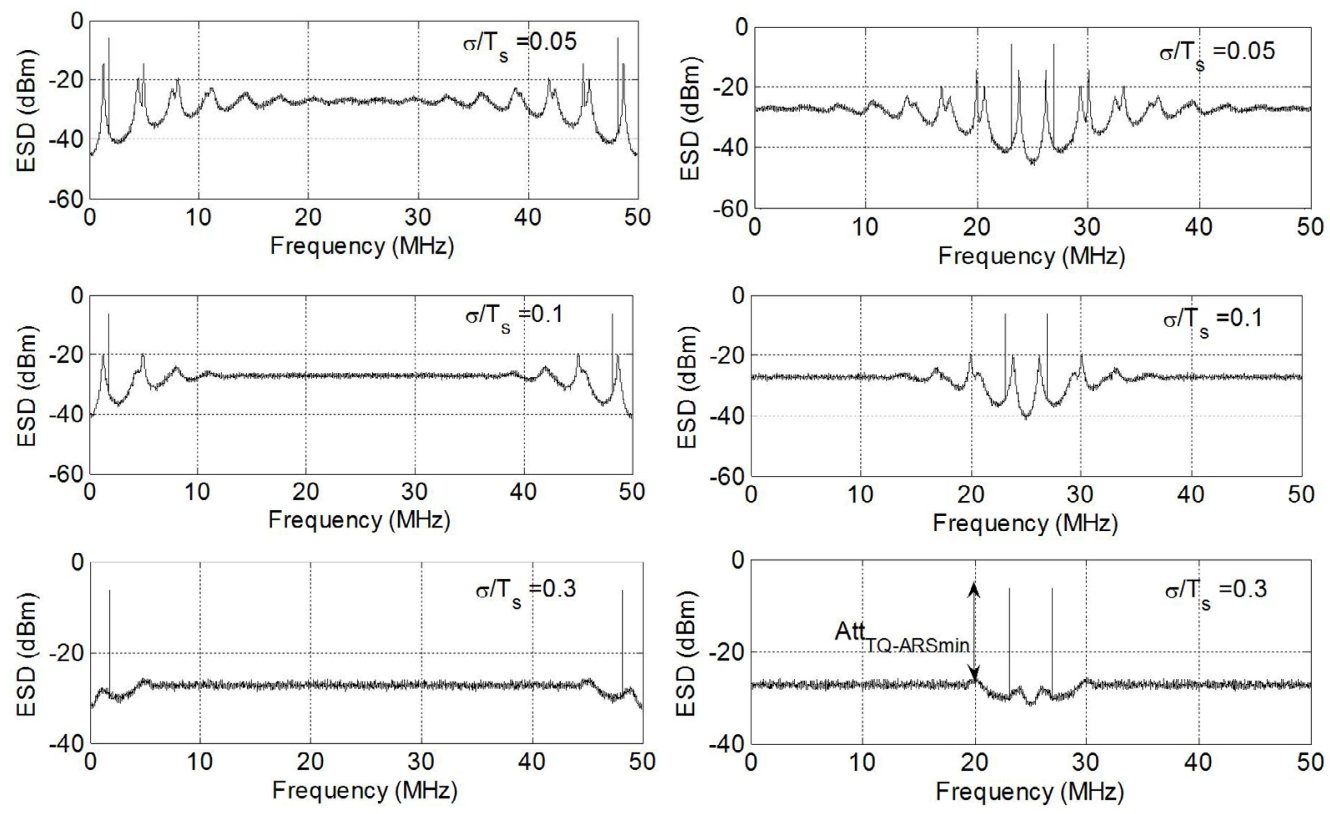

Figure 6: Energy spectrum density function of time-quantized additive randomly sampled signals at a $3.125 \mathrm{MHz}$ mean sampling frequency, for some values of $\sigma / T_{s}$, for $q_{T}=16$, and for $1 \mathrm{MHz}$ single-tone (on the left) and $23 \mathrm{MHz}$ single-tone (on the right) signals. 
the higher the value of $\sigma / T_{s}$ or OSR is, the higher the alias attenuations. The minimum of the alias attenuations, $A t t_{T Q-A R S_{m}}$, denoted as $A t t_{T Q-A R S_{\text {min }}}$, corresponds to the difference between the single-tone signal power and its first replica within $\left[f_{s} / 2,3 f_{s} / 2\right]$. Figure 8 illustrates the minimum attenuation chart that represents some values of $A t t_{T Q-A R S_{\min }}$ versus $\sigma / T_{s}$ for different values of OSR.
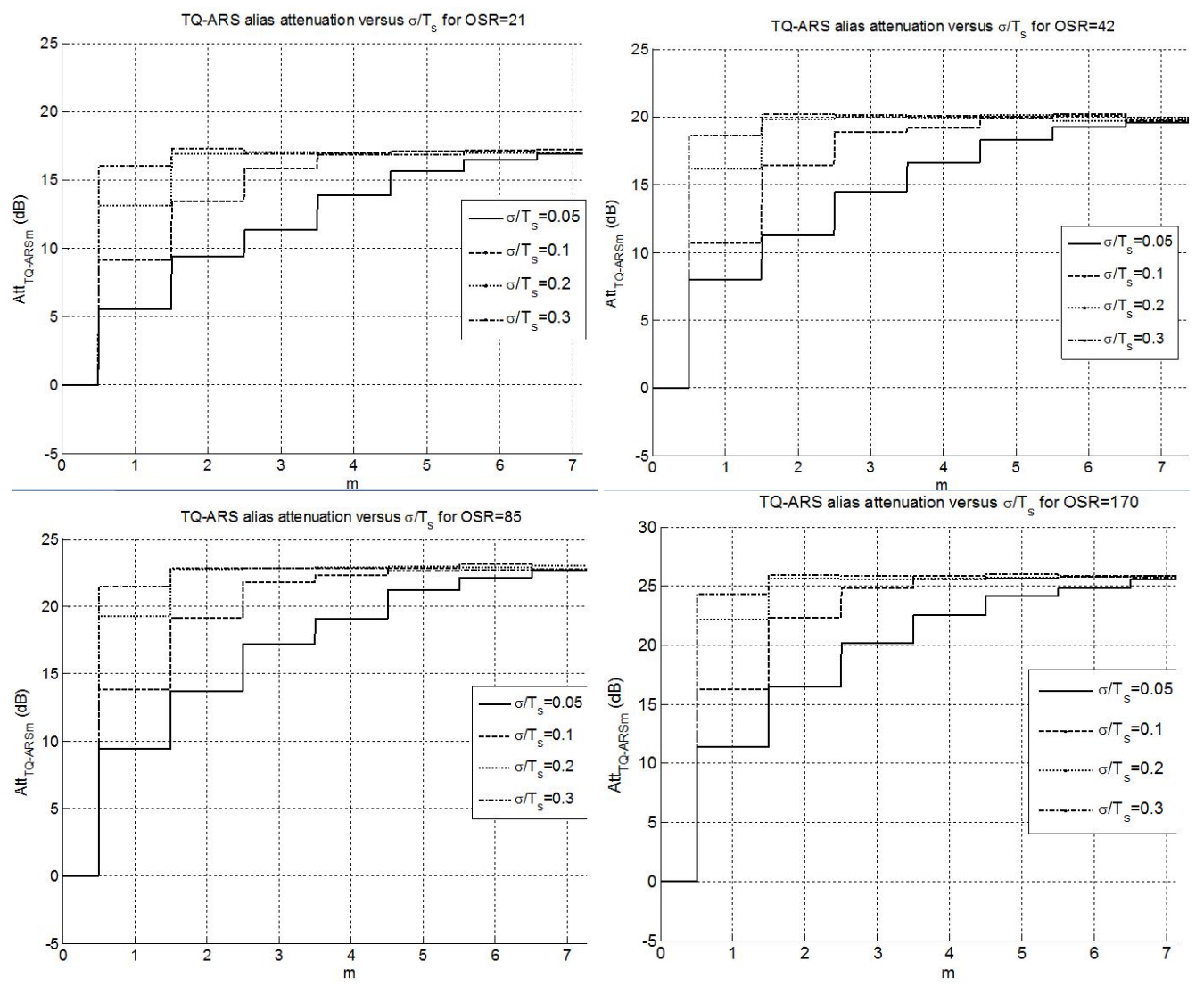

Figure 7: Attenuations' profile $\left\{A t t_{T Q-A R S_{m}}, 1 \leq m \leq\left(q_{T}-1\right) / 2\right\}$ with $q_{T}=16$, obtained for different values of $\sigma / T_{s}$ and OSR.

To valid the proposed spectral analysis tool, the authors compared it with Bilinskis' theoretical results. For random sampling with a finite number of samples, as for the TQ-ARS scheme, Bilinskis introduces the mean ratio of $\lambda$ as in $(35)$ [8],

$$
\lambda=10 \log _{10}\left(\frac{2 N(1-g)-2 g\left(1-g^{N}\right)}{N^{2}(1-g)^{2}}\right)
$$

where $g=\exp \left(-2 \pi^{2} f_{s}^{2} \sigma^{2}\right)=\exp \left(-2 \pi^{2} \sigma^{2} / T_{s}^{2}\right)$ and $N$ is the number of samples. 


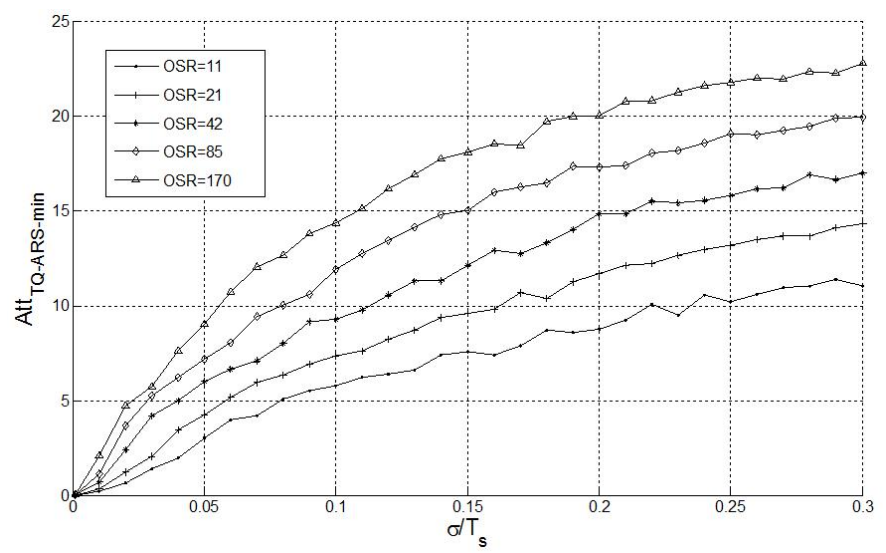

Figure 8: Minimum alias attenuation $A t t_{T Q-A R S_{\min }}$ within $\left[f_{s} / 2,3 f_{s} / 2\right]$ versus $\sigma / T_{s}$ parameter for $q_{T}=16$ and for OSR equal to $11,21,42,85$ and 170 .

The mean ratio, $\lambda$, corresponds in decibels to the opposite of the minimum alias attenuation, $A t t_{T Q-A R S_{\text {min }}}$.

To compare Bilinskis theoretical results with the proposed spectral analysis tool, the authors define $N$ as $2 k_{i n}$ OSR with $k_{i n}$ as the test frequency index related to a represented spectrum over $q_{T} N$. In Figure 8, the curves of $A t t_{T Q-A R S_{\text {min }}}$ give the same values of the opposite values of $\lambda$.

A spectrum analysis and the results of such an attenuation chart as shown in Figure 8 are needed to measure the minimum of the alias attenuation regarding a chosen value of OSR. In the next section, the obtained values of alias attenuation are required to relax the design constraints when adopting the TQ-ARS scheme in a multistandard radio receiver.

\section{Application of TQ-ARS to Relax Design Constraints of a Multistandard Radio Receiver}

The application of random sampling for a multistandard radio receiver is evaluated in this section. The authors propose to take advantage of previous results to design the TQ-ARS-based SDR multistandard radio receiver. The objective is to show how TQ-ARS can relax design constraints on analog baseband stage circuits (AAF, AGC, ADC).

A homodyne architecture is proposed to design an SDR multistandard radio receiver [24]. This topology, that has the advantage of shifting all radio signals processing at baseband, is the most adapted, among analog down-conversion topologies, to multistandard SDR processing. In fact, it reduces the number of discrete components and improves flexibility [17]. Image problem is solved by direct down-conversion. Digital algorithms can be implemented in the digital 
signal processing stage to compensate I/Q mismatch [29] and the DC-offset [30] produced in the homodyne receiver. As shown in Figure 9, the TQ-ARS-based receiver uses a common radio frequency $(\mathrm{RF})$ front-end for multistandard $\mathrm{RF}$ processing. The signal is first received by a multiband antenna [11] and processed by an adequate RF filter selected through an RF switch [34]. Then, the filtered signal is fed to a multiband low-noise amplifier (LNA) [13] and finally downconverted to baseband by quadrature mixers [32].

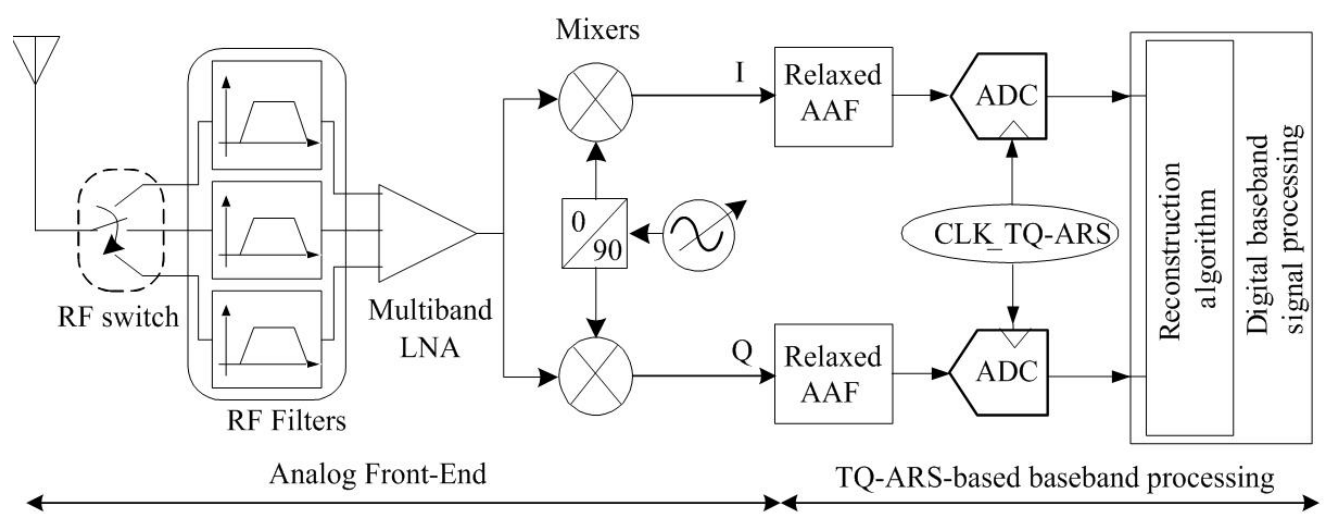

Figure 9: TQ-ARS-based SDR multistandard receiver architecture.

At analog baseband, AAFs are required to sidestep aliases due to sampling. The ADCs have to satisfy constraints in terms of sampling frequency and dynamic range for the chosen standards. In addition, AGCs are generally used to reduce the ADC dynamic range. The TQ-ARS-based SDR multistandard receiver allows the designers to take advantage of alias attenuation to relax constraints on the ADC or AAF and to avoid AGC use. The ADC is driven by a TQ-ARS clock generator (CLK_TQ-ARS), which was proposed by the authors in [24]. A post-conversion baseband signal processing then starts with a reconstruction algorithm to extract uniform samples. The authors in [14] demonstrated that cubic spline reconstruction algorithm offers the best results in terms of dynamic range and complexity.

The low-pass anti-aliasing filter design parameters are the passband frequency $\left(f_{p}\right)$, the rejection frequency $\left(f_{t}\right)$, the maximum in-band attenuation $\left(A_{\max }\right)$, the minimum attenuation $\left(A_{\min }\right)$ at $f_{t}$ and the order $N$. These parameters are deduced as following:

- $f_{p}$ is equal to the channel bandwidth $\mathrm{B}$ with a margin of $30 \%$,

- $f_{t}$, is equal to $f_{s}-B$,

- $A_{\max }$ is equal to $0.3 \mathrm{~dB}$, 
- $A_{\text {min }}$, is given by equation (36) where $N_{b l}$ is the power level of the blocker to attenuate at $f_{t} ; S_{t}$ is the test signal power level given by the standard; $S N R_{\text {out }}$ is the required signal-to-noise ratio; and, $M_{A A F}$ is a margin of 3 $\mathrm{dB}$,

$$
A_{\text {min }}=N_{b l}-S_{t}+S N R_{o u t}+M_{A A F}
$$

- $\mathrm{N}$ is computed under the Butterworth approximation regarding $f_{p}, f_{t}$, $A_{\max }$ and $A_{\min }$.

In this paper, the authors focus on defining the TQ-ARS scheme configuration to relax the AAF design. As shown in Figure 10, the AAF transition band is enlarged by a factor, $q_{T}$, without increasing the sampling frequency and becomes $\left[f_{p}, f_{t_{-} T Q-A R S}\right]$ where $f_{t_{-} T Q-A R S}$ is equal to $q_{T} f_{s}-B$. Moreover, the alias attenuation $A t t_{T Q-A R S \_m i n}$, which is deduced from Figure 8, is subtracted from the $A_{\min }$. Therefore, the $A_{m i n}$ TQ-ARS replaces $A_{\min }$ and given is by (37) instead of (36).

$$
A_{m i n \_T Q-A R S}=N_{b l}-S_{t}+S N R_{o u t}+M_{A A F}-A t t_{T Q-A R S \_m i n}
$$

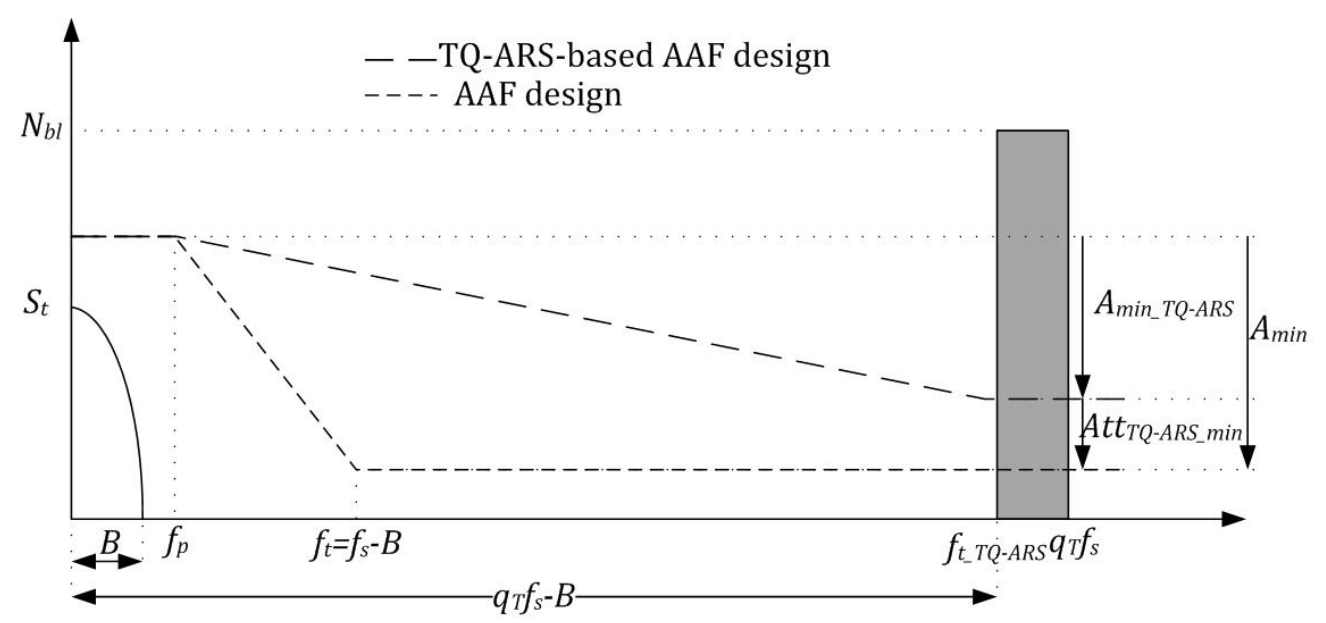

Figure 10: Relaxed anti-aliasing filter design in the case of time-quantized additive random sampling

In order to verify the proposed design results for a multistandard SDR receiver, the authors have chosen the E-GSM (925-960 MHz) [37], UMTS (2110$2170 \mathrm{MHz}$ ) [38], and IEEE802.11a (UNII1-2: 5.15-5.35 GHz, UNII3: 5.725-5.825 $\mathrm{GHz}$ ) standards [39]. Table 1 summarizes the design specifications of the AAF for the case of uniform sampling (US) and TQ-ARS. For both sampling techniques US and TQ-ARS, the OSR values are fixed at 332, 17 and 4 for the EGSM, UMTS and IEEE802.11a standards, respectively. When operating with 
TQ-ARS scheme, the time-quantization factor $q_{T}$ is chosen 16 for the three standards. The blocker levels $N_{b l}$ are deduced from the interferers and blockers profile given by each standard at the rejection frequencies $f_{t}$ and $f_{t_{-} T Q-A R S}$ for US and TQ-ARS techniques, respectively. Hence, for uniform sampling, AAF orders given by the Butterworth approximation are 7, 5 and 4 for E-GSM, UMTS and IEEE802.11a standards, respectively. However, for TQ-ARS technique, a $2^{\text {nd }}$-order AAF is enough for the three standards. Consequently, the TQ-ARS technique allows a lower AAF order without increasing OSR values.

Table 1: E-GSM/UMTS/IEEE802.11a receiver design specifications.

\begin{tabular}{|c|c|c|c|c|c|c|}
\hline & \multicolumn{2}{|c|}{ E-GSM } & \multicolumn{2}{|c|}{ UMTS } & \multicolumn{2}{|c|}{ IEEE802.11a } \\
\hline & $\overline{\mathbf{U S}}$ & TQ-ARS & $\overline{\mathrm{US}}$ & TQ-ARS & $\overline{\mathbf{U S}}$ & TQ-ARS \\
\hline $2 \mathrm{~B}(\mathrm{MHz})$ & \multicolumn{2}{|r|}{0.2} & \multicolumn{2}{|r|}{3.84} & \multicolumn{2}{|r|}{16.6} \\
\hline$f_{p}(\mathrm{MHz})$ & \multicolumn{2}{|r|}{10.79} & \multicolumn{2}{|r|}{10.79} & \multicolumn{2}{|r|}{10.79} \\
\hline OSR & \multicolumn{2}{|r|}{332} & \multicolumn{2}{|r|}{17} & \multicolumn{2}{|r|}{4} \\
\hline$q_{T}$ & - & 16 & - & 16 & - & 16 \\
\hline$f_{s}(\mathrm{MHz})$ & \multicolumn{2}{|r|}{66.4} & \multicolumn{2}{|r|}{66.4} & \multicolumn{2}{|r|}{66.4} \\
\hline$f_{t}(\mathrm{MHz})$ & 66.3 & - & 64.48 & - & 58.1 & - \\
\hline$f_{t \_T Q-A R S}(\mathrm{MHz})$ & - & 1062.3 & - & 1060.48 & - & 1054.1 \\
\hline$N_{b l}(\mathrm{dBm})$ & -23 & -23 & -44 & -44 & -47 & -47 \\
\hline$S_{t}(\mathrm{dBm})$ & \multicolumn{2}{|r|}{-99} & \multicolumn{2}{|r|}{-114} & \multicolumn{2}{|r|}{-62} \\
\hline$S N R_{\text {out }}(\mathrm{dB})$ & \multicolumn{2}{|r|}{9} & \multicolumn{2}{|r|}{-18.2} & \multicolumn{2}{|r|}{26.6} \\
\hline$A_{\min }(\mathrm{dB})$ & 88 & 88 & 54.8 & 54.8 & 44.6 & 44.6 \\
\hline$A t t_{T Q-A R S \_m i n}(\mathrm{~dB})$ & - & 25 & - & 12 & - & 6 \\
\hline$A_{m i n \_T Q-A R S}(\mathrm{~dB})$ & - & 63 & - & 42.8 & - & 38.6 \\
\hline AAF order & 7 & 2 & 5 & 2 & 4 & 2 \\
\hline
\end{tabular}

In the case study of Table 1, the authors relaxed the constraints on the AAF. However, to reduce the constraints on the ADC, the comparison with uniform sampling is made differently. Indeed, in the case of uniform sampling, to obtain a $2^{n d}$-order $\mathrm{AAF}$, the design has to consider 64,8 and 4 times the 66.4 MHz-sampling frequency for E-GSM, UMTS and IEEE802.11a standards, respectively. Therefore, with a $2^{\text {nd }}$-order AAF, the ADC consumes less dynamic power in the case of TQ-ARS than in the case of uniform sampling [6].

The proposed design uses a non-programmable $2^{\text {nd }}$-order filter. In fact, non-programmable filters are passive filters and consume less than active programmable filters, causing noises and distortion. Using a non-programmable $2^{\text {nd }}$-order filter with a fixed passband frequency, which is equal to $10.79 \mathrm{MHz}$, does not filter all interferers and blockers of E-GSM and UMTS standards. This leads to high dynamic ranges which are $96 \mathrm{~dB}$ for E-GSM and $73.8 \mathrm{~dB}$ for UMTS at the ADC input. These constrains can be handled by commercial ADCs [36]. Thus, authors propose the TQ-ARS based SDR multi-standard radio receiver 
which does not require an AGC.

Finally, the alias-free property was tested in a MATLAB simulation environment. A single-tone signal was time-quantized additive randomly sampled, and the quantized output was processed with the cubic spline reconstruction algorithm. Figure 11 shows the same results of the quantized output spectrum after the reconstruction algorithm compared with the uniformly sampled signal spectrum.

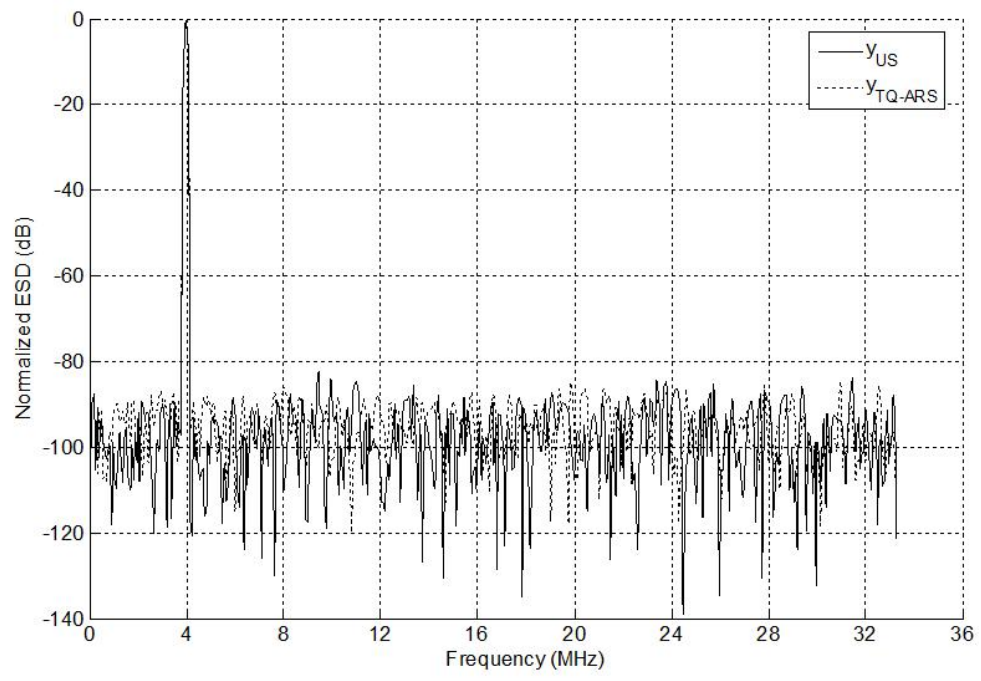

Figure 11: Time-quantized additive randomly sampled signal spectrum after reconstruction $\left(y_{T Q-A R S}\right)$ compared with a uniformly sampled spectrum $\left(y_{U S}\right)$ for a mean sampling frequency or uniform sampling frequency $66.4 \mathrm{MHz}$

\section{Conclusion}

This paper proposed an extension of analytical formulations of random sampling theory. A Fourier transform spectrum estimator was developed to prove that the alias-free condition of an additive random sampling scheme is satisfied in both uniform and Gaussian distributions. For the ARS scheme, the alias attenuation is verified through the formulation of the energy spectrum density. The established formulation considers the deterministic signal and behavior of the initial sampling instant. Nevertheless, random sampling instants disallow practical implementation. Thus, the time-quantized additive random sampling scheme is suggested in the design of a TQ-ARS-based SDR multistandard radio receiver.

The authors also studied existing spectral analysis tools. Limitations were 
discussed in terms of high computational complexity, signal reconstruction requirement or spectrum over $\left[0, f_{s} / 2\right]$ for a mean sampling frequency, $f_{s}$. Therefore, the authors have proposed a spectral analysis tool to represent the spectrum over $\left[0, q_{T} f_{s} / 2\right]$. To obtain a more accurate spectral analysis, the spectrum is presented by computing the spectral analysis average regarding different generated time-quantized additive random sequences. The application of this tool shows that the aliases, Att $T Q-A R S_{m}$, in bands $\left[(2 m-1) f_{s} / 2,(2 m+1) f_{s} / 2\right]$ with $1 \leq m \leq\left(q_{T}-1\right) / 2$ are obtained for different values of the statistical parameter, $\sigma / T_{s}$, and the over-sampling ratio, OSR. Alias attenuation profiles show that the higher the value of $\sigma / T_{s}$ or OSR is, the higher the alias attenuations. The obtained simulation results corroborate Bilinskis' theoretical results. Consequently, the proposed spectrum analysis tool has been validated.

The obtained alias attenuations are considered in the design of a TQ-ARSbased baseband stage for E-GSM / UMTS / IEEE802.11a SDR multistandard homodyne receiver. The ADC is driven by a TQ-ARS clock generator. The design results show that the AAF transition band is increased nearly $q_{T}$ times, without increasing the sampling frequency. The obtained AAF minimum attenuation leads to a relaxed $2^{\text {nd }}$-order filter and to the avoidance of AGC use. To take advantage of the relaxed analog baseband stage, the ADC is followed by a digital baseband signal reconstruction algorithm to extract uniform samples. MATLAB simulations of the TQ-ARS quantized output after reconstruction show the same spectrum as the uniformly sampled signal spectrum.

Future works will focus on computation of the overall performances in terms of signal-to-noise ratio, complexity, power consumption and linearity characteristics of the proposed TQ-ARS-based baseband stage for an E-GSM / UMTS / IEEE802.11a SDR multistandard homodyne receiver. The objective is the implementation of this architecture with at least the same performances as uniform sampling based architecture regarding lower complexity.

\section{ACKNOWLEDGEMENT}

The authors would like to thank the Tunisian-French university cooperation program CMCU for financial support of this research work.

\section{References}

[1] E. Allier, J. Goulier, G. Sicard, A. Dezzani, E. André and M. Renaudin, A 120nm low power asynchronous ADC, Proc. Int. Symp. Low Power Electron. Des., 60-65, 2005.

[2] Y. Artyukh, I. Bilinskis, E. Boole, A. Rybakov and V. Vedin, Wideband RF signal digitizing for high purity spectral analysis, Int. Workshop on Spectral Methods and Multirate Signal Process., 2005. 
[3] P. Augustyniak, Adaptive discrete ECG representation - comparing variable depth decimation and continuous non-uniform sampling, IEEE Comput. Cardiology, 165-168, 2002.

[4] F. Baccelli and P. Brmaud, Palm Probabilities and Stationary Queues, Springer, 1987, ISBN 3-540-96514-9 0-387-96514-9.

[5] A. Baschirotto, Baseband Analog Circuits for Software Defined Radio, Springer, 2008, ISBN 978-1-4020-6537-8.

[6] M. Ben-Romdhane, C. Rebai, A. Ghazel, P. Desgreys, P. Loumeau, Low power data conversion based on non uniform sampling for multistandard receive, IEEE Int. Conf. Des. and Technology of Integrated Syst. in nanoscale era, 261-265, 2009.

[7] R.Z. Bhatti, M. Denneau and J. Draper, Duty cycle measurement and correction using random sampling technique, Proc. 48th IEEE Int. Midwest Symp. Circuits Syst., 1043-1046, 2005.

[8] I. Bilinskis and A. Mikelsons, Randomized Signal Processing, Prentice Hall, 1992, ISBN 0137510748.

[9] D.M. Bland, T.I. Laakso and A. Tarczynski, Analysis of algorithms for nonuniform time discrete Fourier transform, IEEE Int. Symp. Circuits and Syst. ISCAS, 453-456, 1996.

[10] J.D. Coker and A. H. Tewfik, Random sampling strategies in multistatic SAR, IEEE Int. Conf. Acoust., Speech., Signal Process., 2461-2464, 2008.

[11] J. Costantine, K. Kabalan, A. El-Hajj, M. Rammal, New multi-band microstrip antenna design for wireless communications, IEEE Antenna and Propag. Mag., 181-186, 2007.

[12] H. Dammak, M. Ben-Romdhane and K. Grati, Software embedded implementation of real time non uniform sampling filter, IEEE Int. Conf. on Design E Technology of Integrated Systems in Nanoscal Era, 86-89, 2009.

[13] V. K. Dao, Q. D. Bui, C. S. Park, A multi-band $900 \mathrm{MHz} / 1.8 \mathrm{GHz} / 5.2 \mathrm{GHz}$ LNA for reconfigurable radio, IEEE Radio Freq. Integr. Circuits Symp, 69$72,2007$.

[14] H. Farès, M. Ben Romdhane, C. Rebai, Non uniform sampled signal reconstruction for software defined radio applications, IEEE Int. Conf. Signals, Circuits and Syst., 1-6, 2008.

[15] D. Hand and M.S.W. Chen, A non-uniform sampling ADC architecture with embedded alias-free asynchronous filter, Signal Processing for Communications Symposium, Globecom, 3707-3712, 2012. 
[16] S. Kestur, S. Park, K. M. Irick and V. Narayanan, Accelerating the Nonuniform Fast Fourier Transform using FPGAs, IEEE Annual Int. Symp. on Field-Programmable Custom Computing Machines, 19-26, 2010.

[17] J.A. Kilpatrick, R.J. Cyr, E.L. Org, G. Dawe, New SDR architecture enables ubiquitous data connectivity, RF and Microwave Technology for Design Engineers, 32-36, 2006.

[18] A. Maalej, M. Ben-Romdhane, C. Rebai and A. Ghazel, Towards timequantized random sampling for multistandard receiver baseband stage, Int. Conf. on Microelectronics, IEEE, 1-5, 2011.

[19] J.W. Mark and T.D. Todd, A nonuniform sampling approach to data compression, IEEE Trans. Commun., 24-32, 1981.

[20] F. Marvasti, Nonuniform Sampling: Theory and Practice, Kluwer Academic/Plenum Publishers, 2001, ISBN 0-306-46445-4.

[21] E. Masry and A. Vadrevu, A Random sampling estimates of Fourier transforms: antithetical stratified Monte Carlo, IEEE Trans. Signal Process, $\mathbf{5 7}$ (1), 194-204, 2009.

[22] J. Mitola, Software radios: survey, critical evaluation and future directions, IEEE Aerospace and Electron. Syst. Mag., 8, 25-36, 1993.

[23] C. Qin, C.C. Chang and P.L. Tsou, Robust image hashing using nonuniform sampling in discrete Fourier domain, Elsevier, Digital Signal Processing J., 23, 578-585, 2013.

[24] C. Rebai, M. Ben-Romdhane, P. Desgreys, P. Loumeau, A. Ghazel, Pseudorandom signal sampler for relaxed design of multistandard radio receiver, Elsevier Microelectronics Journal, 991-999, 2009.

[25] C.E. Shannon, Communication in the presence of noise, Proc. Inst. of Radio Eng., 10-21, 1949.

[26] H.S. Shapiro and R.A. Silverman, alias-free sampling of random noise, $J$. Soc. Ind.,225-248, 1960.

[27] N. Sharma and T.V. Sreenivas, Sparse signal reconstruction based on signal dependent non-uniform samples, IEEE Int. Conf. on Acoustics, Speech and Signal Processing, 3453-3456, 2012.

[28] O. Shimbo, T. Ohira, K. Nitadori, A generalized formula for the power spectrum of a pulse-modulated signal and its application to PCM transmission problems, Electron. Commun., 1963. 
DESIGN TOOLS FOR RANDOM SAMPLING IN MULTISTANDARD RADIO SIGNAL PROCESSING 143

[29] I. Sohn, E. Jeong, Y.H. Lee,Data-aided approach to I/Q mismatch and DC offset compensation in communication receivers, IEEE Communications Letters,32-36, 2002.

[30] H. Tsurumi, Y. Suzuki, Broadband RF stage architecture for softwaredefined radio in handheld terminal applications, IEEE Communications Magazine, 90-95, 1999.

[31] K. Tzvetkov and A. Tarczynski, On digital filtering of band-limited signals using lower sampling rates, J. of Computers, 5 (10), 1486-1493, 2010.

[32] M.B. Vahidfar, O. Shoaei, F. Svelto, A high dynamic range multi-standard CMOS mixer for GSM, UMTS and IEEE802.11b-g-a applications, IEEE Radio Frequency Integrated Circuits Symposium, 193-196, 2008.

[33] J.J. Wojtiuk, Randomized Sampling for Radio Design, PhD Thesis, University of South Australia, School of Electrical and Information Engineering, 2000 .

[34] S. Yoshimoto, Y. Yamamoto, Y. Takahashi, E. Otsuka, Multi-band RF SAW filter for mobile phone using surface mount plastic package, IEEE Ultrason. Symp, Speech and Signal Processing, 113-118, 2002.

[35] M. Zaare and M. Maymandi-Nejad, A New Power Efficient Wireless NonUniform Adaptive-Sampling ECG Recording System, 21st Iranian Conference on Electrical Engineering, IEEE, 1-5, 2013.

[36] AD9261, 16-Bit, $10 \mathrm{MHz}$ Bandwidth, 30 MSPS to 160 MSPS Continuous Time Sigma-Delta ADC, Analog Devices Inc., 2010, online http://www.analog.com/static/imported-files/data_sheets/AD9261.pdf.

[37] GSM, Radio Transmission and Reception GSM 05.05, ETSI, 1996.

[38] UMTS, UE, Radio Transmission and Reception (FDD), 3GPP TS 25.101, Version 5.2.0 Release 5, ETSI, 2002.

[39] IEEE Standard 802.16, Part 16: Air Interface for Fixed Broadband Wireless Access Systems, IEEE, 2004. 